\title{
Adenosine deaminase negative pleural tuberculosis: a case report
}

\author{
Zachary H. Boggs ${ }^{*} \mathbb{D}$, Scott Heysell, Joshua Eby and Christopher Arnold
}

\begin{abstract}
Background: A pleural fluid adenosine deaminase (ADA) has been used globally to assist in the diagnosis of a tuberculous pleural effusion (TPE) with a notable negative predictive value.

Case presentation: We report a case of a patient with a negative pleural fluid ADA who was found to have culture-positive and biopsy-proven Mycobacterium tuberculosis.

Conclusions: This case shows the importance of pursuing gold standard diagnostic studies when clinical suspicion remains high despite negative preliminary testing. We further describe gaps in research to improve pleural fluid biomarkers for TPE.
\end{abstract}

Keywords: Tuberculosis, Adenosine deaminase, Pleural effusion, IGRA, Case report

\section{Background}

Tuberculosis (TB) is the world's leading killer by a single infectious pathogen. Pleural effusions occur in nearly $5 \%$ of all M. tuberculosis cases [1]. In endemic settings, TB remains the most common cause of a lymphocytic pleural effusion. The gold standard in diagnosis has been through acid fast bacilli (AFB) culture of pleural fluid or through pleural biopsy [2]. Pleural biopsy has been the procedure traditionally used to diagnose a pleural effusion caused by $M$. tuberculosis but carries substantial morbidity. Pleural fluid molecular markers have been developed to forgo invasive testing. Pleural fluid adenosine deaminase (ADA) has a high sensitivity (0.92) and specificity (0.90) for tuberculous pleural effusions (TPE), with a positive likelihood ratio of 9 and a negative likelihood ratio of 0.10 summarized across studies [2-6]. Some have recommended that an ADA level less than $40 \mathrm{IU} / \mathrm{L}$ rules out TPE with its exceptional negative predictive value [2].

\footnotetext{
* Correspondence: zb3ca@virginia.edu

University of Virginia, Charlottesville, VA 22903, USA
}

\section{Case presentation}

We present a case of a human immunodeficiency virusnegative 37-year-old man with 4 months of an intermittent, non-productive cough with associated weight loss (body mass index of 19). He had recently spent 6 weeks in the Amazonian jungle and had traveled to Botswana and India within 2 years of presentation. His brother had been previously treated with active TB. His symptoms progressed with the onset of chest discomfort prompting medical evaluation. A chest $\mathrm{x}$-ray showed a right pleural effusion without other pulmonary nodules (Fig. 1). The initial pleural fluid studies showed an exudative process by Light's criteria and a cell count was not obtained. Bacterial, fungal and AFB cultures were collected, with no elements seen on initial Gram's stain or AFB smear. He was discharged on azithromycin for presumed parapneumonic effusion. He returned to the hospital 6 days later with increased chest pain and fevers. Repeat thoracentesis demonstrated pleural fluid with 1900 white blood cells/ $\mu \mathrm{L}$ (25\% polymorphonuclear leukocytes, $54 \%$ atypical lymphocytes and $21 \%$ lymphocytes). Pleural lactate dehydrogenase was 1121 units/L, glucose $86 \mathrm{mg} / \mathrm{dL}, \mathrm{pH} 7.5$ and total protein $5.0 \mathrm{~g} / \mathrm{dL}$. Repeat pleural fluid bacterial, fungal and AFB cultures (two 


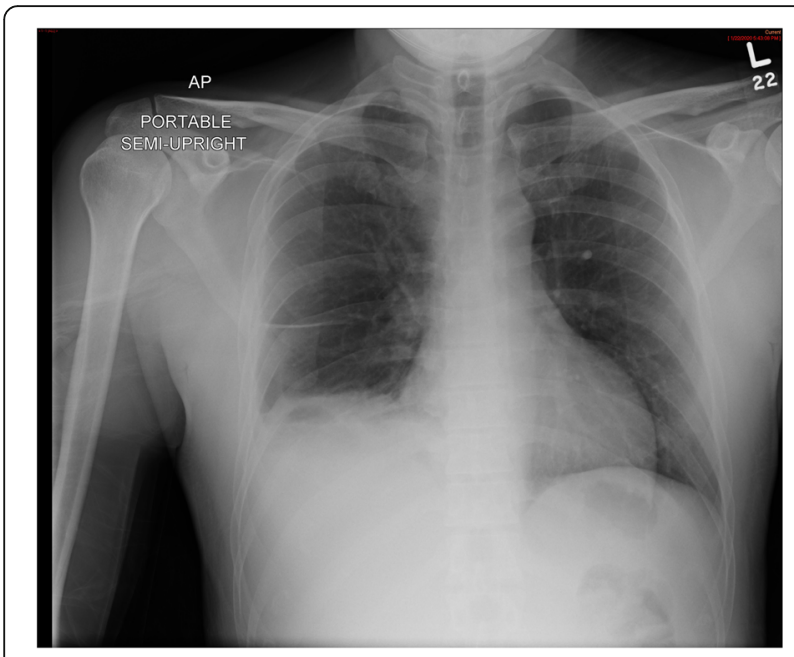

Fig. 1 Chest radiograph of the patient showing a right pleural effusion

slants on Lowenstein-Jensen media, one in liquid media with Mycobacterium Growth Indicator) were obtained and again no organisms were seen on Gram's stain or AFB smear. Nucleic acid amplification testing (NAAT) for $M$. tuberculosis on the pleural sample was negative (Mayo MTBRP). Sputum AFB cultures revealed no growth. Sputum NAAT (Cepheid GeneXpert MTB/RIF) for TB was negative. A pleural fluid ADA (ARUP 3002978) returned at $6.5 \mathrm{IU} / \mathrm{L}$ by quantitative spectrophotometry. Given the very low ADA value, negative NAAT and that cultures of the pleural fluid were preliminarily without growth, alternative diagnoses were considered, and anti-TB therapy was withheld. The otherwise more exhaustive diagnostics included a multiplexed respiratory virus polymerase chain reaction panel, urine antigen studies for Histoplasmosis and Blastomyces species, and autoimmune serologies; all of which were unremarkable. While computed tomography imaging did not show enlarged lymph nodes to suggest lymphoma and pleural cytology did not demonstrate malignant cells, there were a few small calcified supraclavicular lymph nodes and thus given the very low ADA value and lack of alternate diagnosis, a thoracoscopic pleural biopsy was performed. On hospital day 21, his initial pleural fluid cultures grew $M$. tuberculosis, and later his pleural biopsy also grew M. tuberculosis. While awaiting the diagnostic work-up and without anti-TB therapy, he received numerous administrations of antipyretics and continued to lose weight. Once the diagnosis of TB was ultimately made, he was initiated on typical four-drug treatment for drug-susceptible TB. However, he subsequently developed hepatoxicity and required treatment interruption before continuing a triple-drug, pyrazinamide-sparing regimen necessitating an extended total duration. His total hospital course was 30 days, and now has completed treatment. He is clinically improved to near baseline health with a resolved pleural effusion by repeat imaging.

\section{Discussion}

A pleural fluid ADA, given its negative predictive value, is used worldwide to assist in the diagnosis of TPE. AFB cultures often take several weeks to return positive and thoracoscopic biopsy is invasive, holds inherent procedural risks, and is frequently unavailable in TB-endemic settings. Multiple studies have shown that a pleural ADA can aid in TPE diagnosis, while a low level (less than $40 \mathrm{IU} / \mathrm{L}$ ) is effective in ruling out TPE, even in low prevalence areas [5-9]. However, most studies were conducted in high incidence settings and had a high risk for bias [6]. False negative tests have been implicated in early infection as ADA is not active until there is lymphocytic proliferation, however the patient described in this case had symptoms of TB including prominent weight loss and pleurisy for months prior to the pleural fluid ADA testing [10]. Furthermore, advanced age and tobacco use have been shown to cause a lower pleural ADA in the setting of TPE $[11,12]$. The patient was young and a non-smoker and should not have had other reasons to have a blunted ADA response. Common variable immune deficiency could lead to a reduced ADA level, however this patient's IgG levels were tested and normal. Thus, this case demonstrates that ADA should not remain the sole diagnostic test to rule-out TPE when there remains a high suspicion, and this may be particularly problematic in TB endemic settings if the use of ADA as a negative predictor was used to withhold empiric TB treatment.

Despite the incorporation of ADA into diagnostic algorithms for TPE, the diagnosis remains challenging (Table 1). Pleural interferon-gamma release assays (IGRA), which test fluid interferon-gamma production when stimulated with $M$. tuberculosis specific antigens, have been studied in recent years as potential tests to rapidly rule in or rule out a TPE, however meta-analyses have shown variable diagnostic accuracy [14-16]. Nucleic acid amplification tests have revolutionized sputum-based diagnostics for pulmonary TB but are less sensitive for TPE where the bacillary burden is often low [17]. A recent metanalysis has shown that GeneXpert testing has low sensitivity but high specificity in the evaluation of a TPE, thus may have some utility when positive [18]. Xpert MTB/Rif Ultra has been shown to have a high sensitivity in all specimens including cerebrospinal fluid in the diagnosis of TB meningitis, thus has potential for higher yield in pleural fluid compared to conventional Xpert MTB/Rif [20]. Other novel pleural markers are being studied, including interleukin-27, which has been shown in one meta-analysis to be more specific and sensitive than ADA or IGRA testing [19]. 
Table 1 This table summarizes the meta-analyses for multiple biomarkers and rapid tests on pleural fluid to assist in the diagnosis of TPE. ADA = Adenosine Deaminase; IFNy = Interferon Gamma; NAAT = Nucleic Acid Amplification Test; Xpert MTB-Rif = Xpert Mycobacterium tuberculosis Complex and Resistance to Rifampin Assay; IL-27 = Interleukin-27; LR = Likelihood Ratio

\begin{tabular}{lllllll}
\hline Biomarker & Study & Sensitivity & Specificity & +LR & -LR & Summary \\
\hline ADA & Liang et al. 2008 [4] & 0.92 & 0.90 & 9.03 & 0.10 & ADA useful in diagnosis of TPE \\
& Gui et al. 2014 [13] & 0.86 & 0.88 & 6.32 & 0.15 & ADA is relatively accurate \\
& Aggarwal et al. 2019 [6] & 0.92 & 0.90 & 8.92 & 0.09 & Good accuracy regardless of prevalence or setting \\
IFNy & Jiang et al. 2007 [14] & 0.89 & 0.97 & 23.45 & 0.11 & Relatively accurate \\
& Zhou et al. 2011 [15] & 0.75 & 0.82 & 3.49 & 0.24 & Not a useful test \\
\multirow{3}{*}{ NAAT } & Aggarwal et al. 2015 [16] & 0.72 & 0.79 & 3.65 & 0.31 & Not a useful test \\
Xpert MTB-Rif & Pai et al. 2004 [17] & 0.62 & 0.98 & 25.4 & 0.40 & Unclear utility in TPE \\
IL-27 & Sehgal et al. 2016 [18] & 0.51 & 0.99 & 36.7 & 0.49 & Possibly useful when positive \\
\hline
\end{tabular}

Some research groups have considered diagnostic models of multiple pleural biomarkers in combination to accurately diagnose TPE. Ultimately, more research is needed prior to using these tests to make treatment decisions in the absence of AFB culture or pleural biopsy, and pragmatic trials would advance the field beyond the current excess of diagnostic accuracy studies.

\section{Conclusions}

Diagnosing and confirming TPE can be difficult as pleural culture can take weeks and biopsy is invasive or may be unavailable in TB endemic settings. While pleural fluid ADA has been shown previously to have an excellent negative predictive value, we present a case of ADA-negative TPE without risk factors for a falsely low ADA value. In a patient with a high likelihood of TB, a pleural fluid ADA should not be used exclusively to rule-out TPE. Further research should focus on the predictive accuracy of multiple pleural fluid biomarkers and pragmatic trials of $\mathrm{TB}$ treatment initiation.

\section{Abbreviations \\ TPE: Tuberculous pleural effusion; ADA: Adenosine deaminase; TB: Tuberculosis; AFB: Acid fast bacilli; IGRA: Interferon-gamma release assays; IL-27: Interleukin-27; NAAT: Nucleic acid amplification test; IFNY: Interferon gamma; LR: Likelihood ratio; ARUP: Associated regional and university pathologists Lab; Xpert MTB-Rif: Xpert mycobacterium tuberculosis complex} and resistance to rifampin assay

\section{Acknowledgements}

Not applicable.

\section{Authors' contributions}

ZB: original writing and literature review; SH, JE and CA: significant manuscript editing and conceptualization. All authors read, edited, and approved the final version of the manuscript.

\section{Funding}

There were no funding sources for this case report.

\section{Availability of data and materials}

All data generated or analyzed during this study are included in this published article.

\section{Declarations}

Ethics approval and consent to participate

Not applicable.

\section{Consent for publication}

Written informed consent was obtained from the patient for publication of this case report and accompanying images. A copy of the written consent is available for review on request.

\section{Competing interests}

The authors declare that they have no competing interests.

Received: 19 February 2021 Accepted: 3 June 2021

Published online: 15 June 2021

\section{References}

1. Seibert AF, Haynes J, Middleton R, Bass JB. Tuberculous pleural effusion: twenty-year experience. Chest. 1991;99(4):883-6. https://doi.org/10.1378/ chest.99.4.883.

2. Zhai K, Lu Y, Shi HZ. Tuberculous pleural effusion. J Thorac Dis. 2016;8(7): E486-94. https://doi.org/10.21037/jtd.2016.05.87.

3. Piras MA, Gakis C, Budroni M, Andreoni G. Adenosine deaminase activity in pleural effusions: an aid to differential diagnosis. Br Med J. 1978;2(6154): 1751-2. https://doi.org/10.1136/bmj.2.6154.1751-a PubMed PMID: 737480; PubMed Central PMCID: PMC1610017.

4. Liang QL, Shi HZ, Wang K, Qin SM, Qin XJ. Diagnostic accuracy of adenosine deaminase in tuberculous pleurisy: a meta-analysis. Respir Med. 2008;102(5): 744-54. https://doi.org/10.1016/j.rmed.2007.12.007 Epub 2008 Jan 28. Review. PubMed PMID: 18222681

5. Porcel JM, Esquerda A, Bielsa S. Diagnostic performance of adenosine deaminase activity in pleural fluid: a single-center experience with over 2100 consecutive patients. Eur J Intern Med. 2010;21(5):419-23. https://doi. org/10.1016/j.ejim.2010.03.011 Epub 2010 May 1. PubMed PMID: 20816597.

6. Aggarwal AN, Agarwal R, Sehgal IS, Dhooria S. Adenosine deaminase for diagnosis of tuberculous pleural effusion: a systematic review and metaanalysis. PLoS One. 2019;14(3):e0213728. Published 2019 Mar 26. https://doi. org/10.1371/journal.pone.0213728.

7. Ogata Y, Aoe K, Hiraki A, Murakami K, Kishino D, Chikamori K, et al. Is adenosine deaminase in pleural fluid a useful marker for differentiating tuberculosis from lung cancer or mesothelioma in Japan, a country with intermediate incidence of tuberculosis? Acta Med Okayama. 2011;65(4):25963. https://doi.org/10.18926/AMO/46851 PubMed PMID: 21860532.

8. Michot JM, Madec Y, Bulifon S, Thorette-Tcherniak C, Fortineau N, Noël N, et al. Adenosine deaminase is a useful biomarker to diagnose pleural tuberculosis in low to medium prevalence settings. Diagn Microbiol Infect Dis. 2016;84(3):215-20. https://doi.org/10.1016/j.diagmicrobio.2015.11.007 Epub 2015 Nov 10. PubMed PMID: 26707067.

9. Sivakumar P, Marples L, Breen R, Ahmed L. The diagnostic utility of pleural fluid adenosine deaminase for tuberculosis in a low prevalence area. Int J 
Tuberc Lung Dis. 2017;21(6):697-701. https://doi.org/10.5588/ijtld.16.0803 PubMed PMID: 28482965.

10. Valdés L, Pose A, San José E, Martínez Vázquez JM. Tuberculous pleural effusions. Eur J Intern Med. 2003;14(2):77-88. https://doi.org/10.1016/509536205(03)00018-9 PubMed PMID: 12719023

11. Tay TR, Tee A. Factors affecting pleural fluid adenosine deaminase level and the implication on the diagnosis of tuberculous pleural effusion: a retrospective cohort study. BMC Infect Dis. 2013;13:546. Published 2013 Nov 16. https://doi.org/10.1186/1471-2334-13-546.

12. Lee SJ, Kim HS, Lee SH, Lee TW, Lee HR, Cho YJ, et al. Factors influencing pleural adenosine deaminase level in patients with tuberculous pleurisy. Am J Med Sci. 2014;348(5):362-5. https://doi.org/10.1097/MAJ.00000000000002 60 PubMed PMID: 24762755.

13. Gui X, Xiao H. Diagnosis of tuberculosis pleurisy with adenosine deaminase (ADA): a systematic review and meta-analysis. Int J Clin Exp Med. 2014;7(10): 3126-35 Published 2014 Oct 15.

14. Jiang J, Shi HZ, Liang QL, Qin SM, Qin XJ. Diagnostic value of interferongamma in tuberculous pleurisy: a metaanalysis. Chest. 2007;131(4):1133-41. https://doi.org/10.1378/chest.06-2273.

15. Zhou Q, Chen YQ, Qin SM, Tao XN, Xin JB, Shi HZ. Diagnostic accuracy of Tcell interferon- $\gamma$ release assays in tuberculous pleurisy: a meta-analysis. Respirology. 2011;16(3):473-80. https://doi.org/10.1111/j.1440-1843.2011.01 941.x.

16. Aggarwal AN, Agarwal R, Gupta D, Dhooria S, Behera D. Interferon gamma release assays for diagnosis of pleural tuberculosis: a systematic review and meta-analysis. J Clin Microbiol. 2015;53(8):2451-9. https://doi.org/10.1128/ JCM.00823-15 Epub 2015 May 20. Review. PubMed PMID: 25994163; PubMed Central PMCID: PMC4508404.

17. Pai M, Flores LL, Hubbard A, Riley LW, Colford JM Jr. Nucleic acid amplification tests in the diagnosis of tuberculous pleuritis: a systematic review and meta-analysis. BMC Infect Dis. 2004;4(1):6. https://doi.org/10.11 86/1471-2334-4-6 Review. PubMed PMID: 15102325; PubMed Central PMCI D: PMC387423.

18. Sehgal IS, Dhooria S, Aggarwal AN, Behera D, Agarwal R. Diagnostic performance of Xpert MTB/RIF in Tuberculous pleural effusion: systematic review and meta-analysis. J Clin Microbiol. 2016;54(4):1133-6. https://doi. org/10.1128/JCM.03205-15 Epub 2016 Jan 27. PMID: 26818675; PMCID: PMC4809962.

19. Liu Q, Yu YX, Wang XJ, Wang Z, Wang Z. Diagnostic accuracy of interleukin27 between tuberculous pleural effusion and malignant pleural effusion: a meta-analysis. Respiration. 2018;95(6):469-77. https://doi.org/10.1159/0004 86963 Epub 2018 Mar 14. Review. PubMed PMID: 29539604.

20. Huang M, Wang G, Sun Q, Jiang G, Li W, Ding Z, et al. Diagnostic accuracy of Xpert MTB/RIF ultra for tuberculous meningitis in a clinical practice setting of China. Diagn Microbiol Infect Dis. 2021;100(1):115306. https://doi. org/10.1016/j.diagmicrobio.2020.115306 Epub ahead of print. PMID: 33516988

\section{Publisher's Note}

Springer Nature remains neutral with regard to jurisdictional claims in published maps and institutional affiliations.

Ready to submit your research? Choose BMC and benefit from:

- fast, convenient online submission

- thorough peer review by experienced researchers in your field

- rapid publication on acceptance

- support for research data, including large and complex data types

- gold Open Access which fosters wider collaboration and increased citations

- maximum visibility for your research: over $100 \mathrm{M}$ website views per year

At BMC, research is always in progress.

Learn more biomedcentral.com/submissions 